\title{
Constraints on primordial black holes: The importance of accretion
}

\author{
V. De Luca $\odot,{ }^{1}$ G. Franciolini $\odot,{ }^{1,2}$ P. Pani, ${ }^{3,4}$ and A. Riotto ${ }^{1,4}$ \\ ${ }^{1}$ Département de Physique Théorique and Centre for Astroparticle Physics (CAP), \\ Université de Genève, 24 quai Ernest Ansermet, CH-1211 Geneva, Switzerland \\ ${ }^{2}$ Instituto de Física Teórica UAM-CSIC, Universidad Autónoma de Madrid, \\ Cantoblanco, Madrid, 28049 Spain \\ ${ }^{3}$ Dipartimento di Fisica, Sapienza Universit di Roma, Piazzale Aldo Moro 5, 00185 Roma, Italy \\ ${ }^{4}$ INFN, Sezione di Roma, Piazzale Aldo Moro 2, 00185 Roma, Italy
}

(Received 3 April 2020; revised 5 May 2020; accepted 20 July 2020; published 5 August 2020)

We consider the constraints on the fraction of dark matter in the universe in the form of primordial black holes taking into account the crucial role of accretion which may change both their mass and mass function. We show that accretion may drastically weaken the constraints at the present epoch for primordial black holes with masses larger than a few solar masses.

DOI: 10.1103/PhysRevD.102.043505

\section{INTRODUCTION}

The detection of gravitational waves generated by the merger of black holes [1-4] has given rise to a renewed interest in the idea that primordial black holes (PBHs) could compose a fraction (or all) of the dark matter (DM) [5] (see Refs. [6-8] for reviews).

In order to firmly assess if $\mathrm{PBHs}$ with a given mass might contribute to a significant fraction of the DM, a careful investigation should be performed to understand whether the current observational constraints on the $\mathrm{PBH}$ abundance-usually parametrized by the fraction $f_{\mathrm{PBH}} \equiv$ $\Omega_{\mathrm{PBH}} / \Omega_{\mathrm{DM}}$ at the present epoch [8]—apply around that mass.

It goes without saying that understanding what physical phenomena can alter such constraints, either strengthening or weakening them, is of utmost importance. For instance, a large spatial clustering of PBHs might in principle help in avoiding microlensing constraints [9], even though one should take into account that PBHs are initially not clustered (in the absence of primordial non-Gaussianity) [10-13].

PBH mergers and accretion can also have an impact on the PBH bounds. While only a tiny fraction of PBHs detectable through their coalescence have experienced a previous merger event [14], PBHs may efficiently accrete during the cosmic history [15]. In particular, if they do not represent the only DM component, $\mathrm{PBHs}$ may accrete a DM halo, thus increasing their gravitational potential and the ordinary gas accretion [16].

For sufficiently massive PBHs, accretion may occur at super-Eddington rates up to the reionization epoch [14-16]. The mass distribution of PBHs at low redshift is therefore different from the one at high redshift. This implies that limits on the current $\mathrm{PBH}$ abundance, which are expressed in terms of the present $\mathrm{PBH}$ mass values, must be properly revisited. For example, the CMB temperature and polarization fluctuations are sensitive to the energy injection up to redshift $z \sim 300[16,17]$. In the presence of significant accretion up to much lower redshifts, the PBH masses and their distribution measured today (albeit indirectly) do not coincide with those at redshifts $z \sim(300 \div 600)$ when the energy deposition has the largest effect on the CMB.

The goal of this paper is to highlight the significant role played by the phenomenon of accretion in setting the observational limits on the current PBHs abundance. We therefore proceed to briefly describe the main features of accretion onto PBHs and then investigate its impact on the current constraints on $f_{\mathrm{PBH}}$.

\section{ACCRETION ONTO PBHs}

Once PBHs are produced, for instance due to the collapse of sizable overdensities in the radiation-dominated epoch, their evolution may be significantly affected by accretion during the cosmic history $[16,18]$. The physics of accretion is very complex, since the accretion rate and the geometry of the accretion flow are intertwined, and they are both crucial in determining the evolution of the PBH mass.

A PBH of a given mass $M$ immersed in the intergalactic medium can accrete baryonic matter at the Bondi-Hoyle rate given by [19]

$$
\dot{M}_{\mathrm{B}}=4 \pi \lambda m_{H} n_{\mathrm{gas}} v_{\mathrm{eff}} r_{\mathrm{B}}^{2},
$$

where $r_{\mathrm{B}}=G M / v_{\mathrm{eff}}^{2}$ is the Bondi-Hoyle radius, $n_{\mathrm{gas}}$ is the hydrogen gas number density, and $v_{\text {eff }}=\sqrt{v_{\text {rel }}^{2}+c_{s}^{2}}$ is the $\mathrm{PBH}$ effective velocity, defined in terms of the $\mathrm{PBH}$ relative velocity $v_{\text {rel }}$ with respect to the gas with sound speed $c_{s}$. The accretion parameter $\lambda$ takes into account the gas 
viscosity, the Hubble expansion, and the coupling of the gas to the CMB radiation through Compton scattering. Its explicit expression depends on redshift, ionization fraction $x_{e}$, PBH mass and effective velocity, and is given by [14-16]

$$
\lambda=\exp \left(\frac{9 / 2}{3+\hat{\beta}^{0.75}}\right) x_{\mathrm{cr}}^{2},
$$

in terms of the gas viscosity parameter

$$
\begin{aligned}
\hat{\beta}= & \left(\frac{M}{10^{4} M_{\odot}}\right)\left(\frac{1+z}{1000}\right)^{3 / 2}\left(\frac{v_{\text {eff }}}{5.74 \mathrm{~km} \mathrm{~s}^{-1}}\right)^{-3} \\
& \times\left[0.257+1.45\left(\frac{x_{e}}{0.01}\right)\left(\frac{1+z}{1000}\right)^{5 / 2}\right]
\end{aligned}
$$

and the sonic radius

$$
x_{\mathrm{cr}} \equiv \frac{r_{\mathrm{cr}}}{r_{\mathrm{B}}}=\frac{-1+(1+\hat{\beta})^{1 / 2}}{\hat{\beta}} .
$$

If PBHs do not comprise the totality of DM in the Universe, one has to consider the additional presence of a dominant DM component which forms, around each $\mathrm{PBH}$, a dark halo of mass [20,21]

$$
M_{h}(z)=3 M\left(\frac{1+z}{1000}\right)^{-1},
$$

which grows with time as long as the $\mathrm{PBH}$ does not interact with others. While direct accretion of DM is negligible for the PBH evolution [16], the effect of this DM clothing is to enhance the gas accretion rate, acting in this way as a catalyst. The characteristic halo radius, assuming a power law density profile $\rho \sim r^{-\alpha}[16,21,22]$, is given by

$$
r_{h}=0.019 \mathrm{pc}\left(\frac{M}{M_{\odot}}\right)^{1 / 3}\left(\frac{1+z}{1000}\right)^{-1},
$$

and has to be confronted with the Bondi radius by introducing the parameter

$$
\kappa \equiv \frac{r_{\mathrm{B}}}{r_{h}}=0.22\left(\frac{1+z}{1000}\right)\left(\frac{M_{h}}{M_{\odot}}\right)^{2 / 3}\left(\frac{v_{\mathrm{eff}}}{\mathrm{km} \mathrm{s}^{-1}}\right)^{-2} .
$$

In the regime $\kappa \geq 2$ the dark halo behaves as a point mass and the accretion rate will be the same as one for a PBH of mass $M_{h}$. In the opposite regime, $\kappa<2$, one can introduce corrections to the naked case by modifying the accretion parameter as [20]

$$
\hat{\beta}^{h} \equiv \kappa^{\frac{p}{1-p}} \hat{\beta}, \quad \lambda^{h} \equiv \bar{\Upsilon}^{\frac{p}{1-p}} \lambda\left(\hat{\beta}^{h}\right), \quad r_{\mathrm{cr}}^{h} \equiv\left(\frac{\kappa}{2}\right)^{\frac{p}{1-p}} r_{\mathrm{cr}},
$$

where $p=2-\alpha$ and

$$
\bar{\Upsilon}=\left(1+10 \hat{\beta}^{h}\right)^{\frac{1}{10}} \exp (2-\kappa)\left(\frac{\kappa}{2}\right)^{2}
$$

One can normalize the Bondi-Hoyle accretion rate in terms of the Eddington one,

$$
\dot{M}_{\mathrm{Edd}}=1.44 \times 10^{17}\left(\frac{M}{M_{\odot}}\right) \mathrm{g} \mathrm{s}^{-1},
$$

by introducing the dimensionless rate

$\dot{m} \equiv \frac{\dot{M}_{\mathrm{B}}}{\dot{M}_{\mathrm{Edd}}}=0.023 \lambda\left(\frac{1+z}{1000}\right)\left(\frac{M}{M_{\odot}}\right)\left(\frac{v_{\mathrm{eff}}}{5.74 \mathrm{~km} \mathrm{~s}^{-1}}\right)^{-3}$,

such that the mass evolution equation takes the more compact form (see, e.g., Refs. [14,23])

$$
\dot{M} \sim 0.002 \dot{m}(M)\left(\frac{M}{10^{6} M_{\odot}}\right) M_{\odot} \mathrm{yr}^{-1} .
$$

The dimensionless baryonic accretion rate $\dot{m}$ carries all the information on the geometry and efficiency of the accretion process. For example, if the angular momentum carried by the baryonic infalling material is sufficiently high, a thin accretion disk forms around the PBH. Details on that can be found in Ref. [14] and references therein.

From Eq. (12), the typical accretion timescale is $\tau_{\mathrm{ACC}}=$ $\tau_{\text {Salp }} / \dot{m}$, where $\tau_{\text {Salp }}=4.5 \times 10^{8} \mathrm{yr}$ is the Salpeter time. For redshifts $z \gtrsim 30$ the accretion timescale is much longer than the age of the Universe at that epoch, and therefore there is not enough time for the accretion process to play a significant role in the PBH mass evolution. Depending on the PBH masses, after $z \sim 30$ accretion may have an important impact, reaching also super-Eddington values (see Fig. 4 in Ref. [14]).

At smaller redshifts, uncertainties in the accretion model come into play. While local feedbacks are not relevant for the range of $\mathrm{PBH}$ masses of our interest [16], global feedbacks from the radiation emitted by the gas accreting onto the PBHs, along with preheating effects from x-ray backgrounds [24], may heat and ionize the intergalactic medium, leading to sensible changes in its temperature. Moreover, with the beginning of structure formation, one expects the fall of a large part of the $\mathrm{PBH}$ population into the gravitational potential wells of the large-scale structures, leading to an increase of the $\mathrm{PBH}$ peculiar velocities, see in particular Ref. [25] for a recent analysis. This effect, together with reionization and global feedbacks, may lead to a decrease of the accretion rate [16,26-29]. Owing to the uncertainties in the modeling of accretion onto PBHs at redshift $z \sim 10$, we have decided to consider three different 


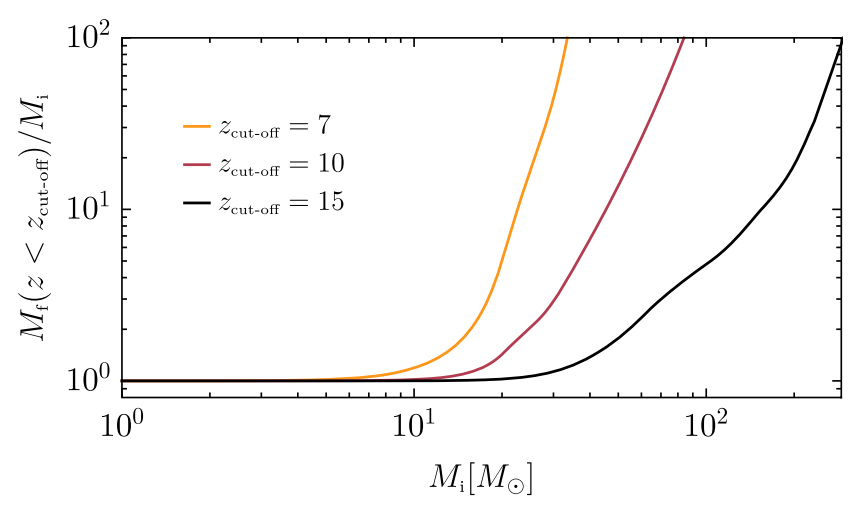

FIG. 1. Final mass $M_{\mathrm{f}}$ at a given redshift $z$ obtained from the evolution of an initial mass $M_{\mathrm{i}}$ in the various scenarios with cutoff redshift $z_{\text {cut-off }}=15,10$ and 7 .

cutoff points and assume that mass accretion becomes negligible after at $z_{\text {cut-off }} \simeq 15,10$ and 7 . The first value has been chosen to show a moderate effect of accretion, the second value corresponds to model I of Ref. [14] and we consider it the most realistic choice, whereas the third value considers the case in which accretion onto PBHs is significant at a relatively smaller redshift, as discussed in Ref. [16].

The accretion-driven evolution of the mass is shown in Fig. 1. For initial masses $M_{\mathrm{i}}$ smaller than a few solar masses, depending on $z_{\text {cut-off }}$, accretion does not have a noticeable impact on the PBHs mass, which remains the same up to the present time. For higher masses, instead, the accretion plays an important role, leading to a final mass $M_{\mathrm{f}}$ at the present epoch which can be various orders of magnitude larger than the initial one.

Accretion has also the additional effect of changing the PBH mass distribution with redshift. We define the mass function $\psi(M, z)$ as the fraction of PBHs with mass in the interval $(M, M+\mathrm{d} M)$ at redshift $z$. For an initial $\psi\left(M, z_{\mathrm{i}}\right)$ at formation redshift $z_{\mathrm{i}}$, its evolution is governed by [14]

$$
\psi\left(M_{\mathrm{f}}(M, z), z\right) \mathrm{d} M_{\mathrm{f}}=\psi\left(M, z_{\mathrm{i}}\right) \mathrm{d} M,
$$

where $M_{\mathrm{f}}(M, z)$ is given in Fig. 1. When accretion is present, also the value of $f_{\mathrm{PBH}}$ depends on the redshift as ${ }^{1}$

$$
\begin{aligned}
f_{\mathrm{PBH}}(z) & =\frac{\rho_{\mathrm{PBH}}}{\left(\rho_{\mathrm{DM}}-\rho_{\mathrm{PBH}}\right)+\rho_{\mathrm{PBH}}} \\
& =\frac{\langle M(z)\rangle}{\left\langle M\left(z_{\mathrm{i}}\right)\right\rangle\left(f_{\mathrm{PBH}}^{-1}\left(z_{\mathrm{i}}\right)-1\right)+\langle M(z)\rangle},
\end{aligned}
$$

defined in terms of the average mass

\footnotetext{
${ }^{1}$ We assume here for simplicity a nonrelativistic dominant DM component, whose energy density scales as the inverse of the volume.
}

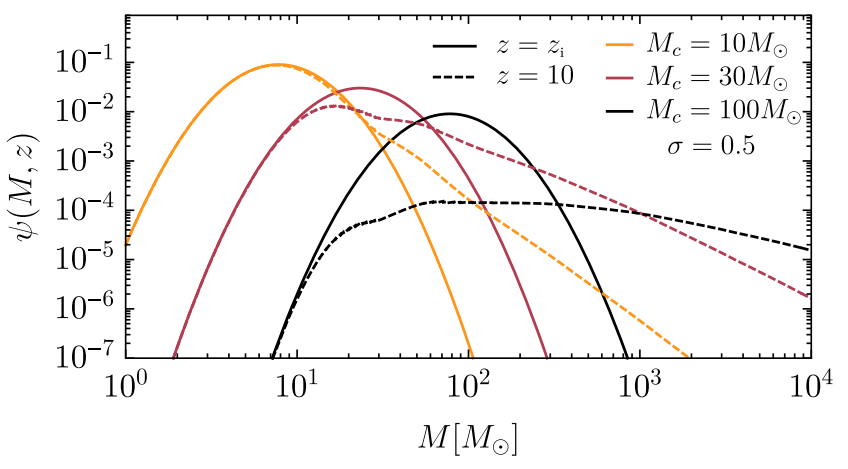

FIG. 2. Evolution of the mass function from formation (solid lines) to redshift $z=10$ (dashed lines), for an initial lognormal shape with width $\sigma=0.5$ at different values of $M_{c}$.

$$
\langle M(z)\rangle=\int \mathrm{d} M M \psi(M, z)
$$

One can consider several initial shapes for the mass function at high redshift, depending on the details of the formation mechanism. Often in the literature an initial sharp monochromatic population is considered, with a constant reference peak mass $M_{c}$. Accretion has the effect of shifting such a peak. For more physically motivated scenarios, one can consider an initial extended mass function given by a lognormal shape with width $\sigma$,

$$
\psi\left(M, z_{\mathrm{i}}\right)=\frac{1}{\sqrt{2 \pi} \sigma M} \exp \left(-\frac{\log ^{2}\left(M / M_{c}\right)}{2 \sigma^{2}}\right),
$$

properly normalized to unity and whose evolution with time is shown in Fig. 2. As one can see, as $M_{c}$ increases, the evolved mass function becomes increasingly flatter with a high-mass tail orders of magnitude above its corresponding value at formation.

The evolution of the abundance $f_{\mathrm{PBH}}$ in the case of a monochromatic mass function can be read off Fig. 1, as

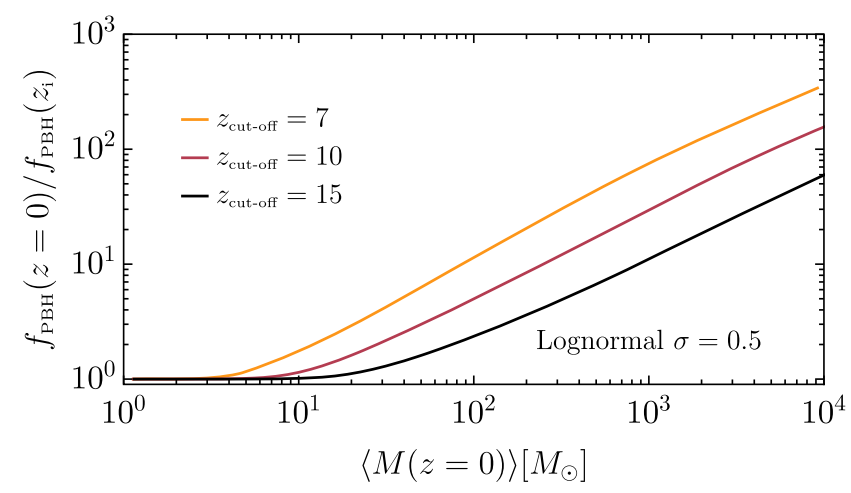

FIG. 3. Ratio between the initial and late time PBH abundance depending on $\langle M(z=0)\rangle$ for an initial lognormal mass function with $\sigma=0.5$, away from the saturation happening when $f_{\mathrm{PBH}}$ approaches unity. 
$f_{\mathrm{PBH}}(z) / f_{\mathrm{PBH}}\left(z_{\mathrm{i}}\right) \sim M_{\mathrm{f}}(z) / M_{\mathrm{i}}$ as long as the abundance is not close to unity. In a similar manner, the corresponding evolution in the case of a lognormal mass function is plotted in Fig. 3 as a function of $\langle M(z=0)\rangle$.

\section{RESULTS}

Detailed investigations on the current observational constraints on the PBH abundance have been performed in the literature (see, e.g., Ref. [8] for a recent review on the topic). In the range of masses affected by accretion, the most important constraints come from lensing, dynamical processes, formation of structures, and accretion related phenomena. The lensing bounds include those from supernovae [30], the MACHO and EROS experiments [31,32], ICARUS (I) [33] and radio [34] observations. They all consider lensing sources at low redshift $z \ll z_{\text {cut-off }}$. Dynamical constraints involve disruption of wide binaries [35], and survival of star clusters in Eridanus II [36] and Segue I [37] at small redshifts. Bounds also arise by observations of the Lyman- $\alpha$ forest at redshift before $z \approx 4$ [38]. Other constraints involve bounds from Planck data on the CMB anisotropies induced by $\mathrm{x}$ rays emitted by spherical or disk (Planck D) $[17,26]$ accretion at high redshifts or bounds on the observed number of $\mathrm{x}$ rays (Xr) [39,40] and $\mathrm{x}$-ray binaries (XrB) at low redshifts [41]. Additional constraints on the primordial abundance can also be set by the LIGO-Virgo observations [42]. A comprehensive plot of all the constraints is shown in Fig. 10 of Ref. [8] for the choice of a monochromatic mass function, while in the same reference the extension to broader mass functions has been obtained following the procedures outlined in Refs. [43,44].

However, these constraints are standardly expressed in terms of the maximum fraction ${ }^{2} f_{\mathrm{PBH}}$ of PBHs allowed in a given mass range; these quantities refer to the present epoch, without taking into account the evolution of the PBH mass due to accretion, whose effects on the constraints are presented in the following. Motivated by the fact that the current LIGO-Virgo observations are at low redshifts, we have decided to present our results in terms of the values of $f_{\mathrm{PBH}}(z=0)$ and $\langle M(z=0)\rangle$ today. Of course, for future data, for instance from the Einstein Telescope [45], the corresponding values can be easily evaluated for a given initial mass function at the redshift of interest.

For simplicity, for a given $\mathrm{PBH}$ mass range, we consider only the most stringent constraint. Following the prescription described in Ref. [43], we estimate the bound on the fraction $f_{\mathrm{PBH}}\left(z_{\mathrm{e}}\right)$ of PBHs as DM at the redshift $z_{\mathrm{e}}$ of a given experiment from

\footnotetext{
${ }^{2}$ The constraints are in fact sensitive to the $\mathrm{PBH}$ number density $n_{\mathrm{PBH}}$ and, when translating the bounds in terms of $f_{\mathrm{PBH}}$, one has to be careful in properly accounting for the accretion effects on an experiment-by-experiment basis.
}

$$
f_{\mathrm{PBH}}\left(z_{\mathrm{e}}\right) \lesssim\left(\int_{M_{\min }\left(z_{\mathrm{e}}\right)}^{M_{\max }\left(z_{\mathrm{e}}\right)} \mathrm{d} M \frac{\psi\left(M, z_{\mathrm{e}}\right)}{f_{\max }\left(M, z_{\mathrm{e}}\right)}\right)^{-1},
$$

where $M_{\min }\left(z_{\mathrm{e}}\right)$ and $M_{\max }\left(z_{\mathrm{e}}\right)$ identify the range of masses affected by the given constraint, and $f_{\max }\left(M, z_{\mathrm{e}}\right)$ represents the maximum allowed fraction for a monochromatic mass function at the redshift of the experiment $[8,43]$.

Thus, for a given constraint $f_{\max }\left(M, z_{\mathrm{e}}\right)$ obtained neglecting accretion, we can compute the above integral by taking into account the evolution of the mass function from formation to $z_{\mathrm{e}}$ through Eq. (13). Finally, the bound $f_{\mathrm{PBH}}\left(z_{\mathrm{e}}\right)$ is mapped to $f_{\mathrm{PBH}}(z=0)$ using Eq. (14). With this procedure one gets the bound on the present fraction of PBHs as DM $f_{\mathrm{PBH}}(z=0)$ for a given $\langle M(z=0)\rangle$ as shown in Fig. 4 for a monochromatic (left panel) and a lognormal (right panel) mass function. The various values of $\langle M(z=0)\rangle$ have been calculated by varying $M_{c}$.

We choose to plot a single envelope resulting from the most stringent bound for any value of $\langle M(z=0)\rangle$, while the labels identify the corresponding experiment dominating each portion of the graphs. Also, we compare the results in the scenarios with $z_{\text {cut-off }}=15,10$ and 7 with the original constraints which neglect the effect of accretion. It is worthwhile to stress that the bound Planck D from the CMB is characterized by $z_{\mathrm{e}} \gtrsim 300$ and therefore has to be computed at that redshift using the initial mass function, as standardly done in the literature. As for the other experiments, for which lower values of $z_{\mathrm{e}}$ are involved, the bounds have to be estimated with the evolved mass function.

As one can appreciate from Fig. 4, the observational bounds are drastically weakened at the present epoch, which is of particular importance when asking the question whether a given merger event is consistent with the hypothesis that the black holes are of primordial origin. In particular, the relaxation of the bounds for a monochromatic mass function depends solely on the shift of the peak of the distribution and the corresponding evolution of $f_{\mathrm{PBH}}(z)$. In this case, since the bounds in Fig. 4 refer to the average $\mathrm{PBH}$ mass at the present epoch, only the constraints obtained with observations at high redshifts are affected (i.e., Planck D in the left panel of Fig. 4). Note that, even if the constraints obtained with observations at small redshifts are unaffected in the case of a monochromatic distribution, they still refer to a different mass $\langle M(z=0)\rangle$ relative to the case in which accretion is absent, for which $\langle M(z=0)\rangle=M_{c}$. Therefore, if one wants to constrain the PBH formation scenario when accretion is present, and in particular the parameter $M_{c}$, one needs to use the mapping between $\langle M(z=0)\rangle$ and $M_{c}$. On the other hand, for extended distributions (right panel of Fig. 4), the relaxation of the constraints depends also on the more complex evolution of $\psi(M, z)$, which affects not only the bounds at high redshifts, but also those at low redshifts, since the latter are inferred assuming a broader distribution. 

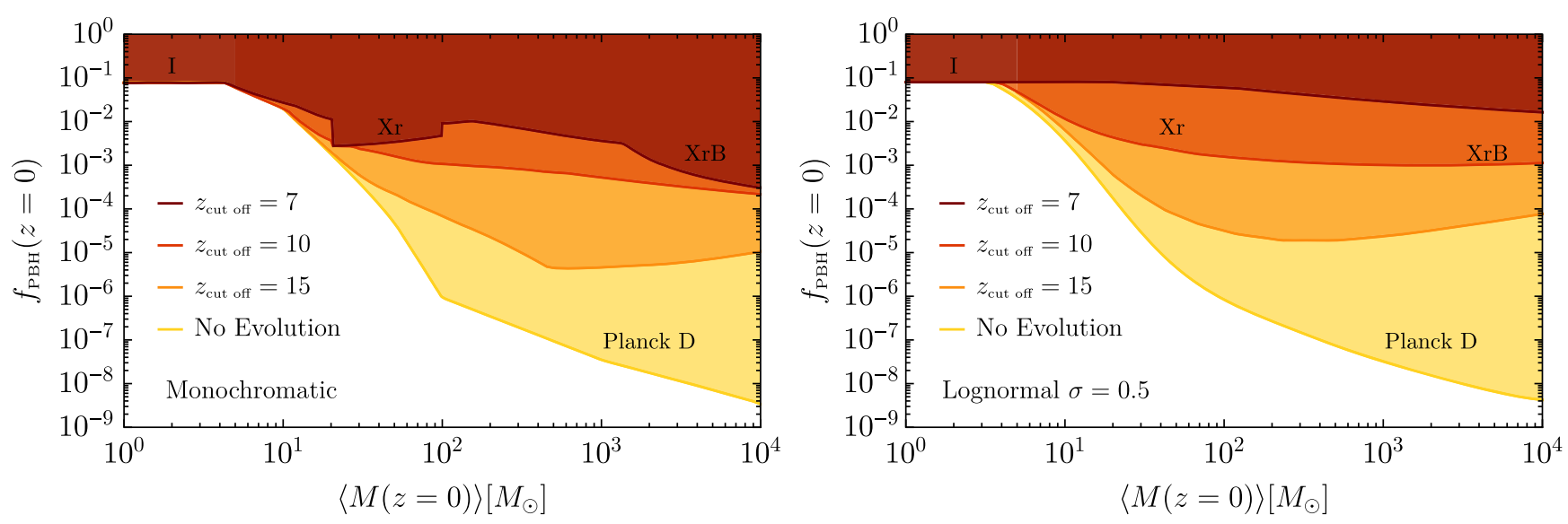

FIG. 4. Combined constraints on the fraction of DM composed by PBHs today in terms of $\langle M(z=0)\rangle$ for the different accretion models corresponding to $z_{\text {cut-off }}=15,10$ and 7 compared to the case in which accretion is neglected ("No Evolution"). Left: monochromatic case. Right: lognormal mass function with width $\sigma=0.5$ at formation.

\section{CONCLUSIONS}

We have described how accretion onto PBHs may change the interpretation of the observational bounds on the current fraction of PBHs in DM for a given mass range. Our goal was not to perform a comprehensive study, but just to show the crucial impact of $\mathrm{PBH}$ accretion on current bounds. We have assumed an accretion model valid for isolated PBHs. If the latter form a binary at $z \gtrsim 30$ [6], the effects of the binary for the mass accretion rate should be taken into account [14]. Since the overall fraction of PBH in binaries is $\sim 10^{-2} f_{\mathrm{PBH}}^{16 / 37}$ [46], this effect is negligible for our bounds, except possibly for those coming from mergers [47].

Our findings are relevant in the context of the origin of the black hole mergers observed by current gravitationalwave interferometers. Indeed, in the mass range $(10 \div$ $100) M_{\odot}$ accretion can uplift the existing upper limits on $f_{\mathrm{PBH}}(z=0)$ by several orders of magnitude. The effect of accretion on PBHs is intrinsically redshift dependent, so it would be interesting to investigate the consequences of our results for the forecasts of future experiments, like the Einstein Telescope, which will probe higher redshifts and higher PBH masses. It would be also interesting to fully assess the impact of accretion onto the $\mathrm{PBH}$ merger rate and on the corresponding bounds from LIGO-Virgo observations [42] when accounting for DM clothing. We will investigate this issue in a separate publication [47].

Our analysis can be improved along several ways. One is certainly having a better knowledge of the impact of large-scale structures, reionization and global thermal feedbacks onto the PBH accretion; another one is a better characterization of accretion for values of the PBH fraction close to unity, where our assumption of DM clothing ceases to be correct. This would be particularly relevant in order to understand the fate of $f_{\mathrm{PBH}}$ in the case of strong accretion, since as one can see from Eq. (14), when $\langle M(z)\rangle \gg\left\langle M\left(z_{\mathrm{i}}\right)\right\rangle / f_{\mathrm{PBH}}\left(z_{\mathrm{i}}\right), \quad f_{\mathrm{PBH}}(z)$ can dynamically approach unity, even if the initial fraction of PBHs in DM is negligible. Finally, a more detailed characterization of accretion would be instrumental to assess if relatively light PBHs may play a role in explaining the supermassive black holes observed at $z \gtrsim 6$ [48].

\section{ACKNOWLEDGMENTS}

We thank B. Carr and M. Sasaki for discussions. V. D. L., G. F. and A. R. are supported by the Swiss National Science Foundation (SNSF), project The Non-Gaussian Universe and Cosmological Symmetries, Project No. 200020178787. G. F. would like to thank the Instituto de Fisica Teorica, Universidad Autonoma de Madrid-Consejo Superior de Investigaciones Cienticas (IFT UAM-CSIC) in Madrid for its support via the Centro de Excelencia Severo Ochoa Program under Grant No. SEV-2012-0249. P.P. acknowledges financial support provided under the European Union's H2020 ERC, Starting Grant Agreement No. DarkGRA-757480, under the MIUR PRIN and FARE programs (GW-NEXT, CUP: B84I20000100001), and support from the Amaldi Research Center funded by the MIUR program "Dipartimento di Eccellenza" (CUP: B81I18001170001). 
[1] B. P. Abbott et al. (LIGO Scientific and Virgo Collaborations), Phys. Rev. Lett. 116, 061102 (2016).

[2] B. P. Abbott et al. (LIGO Scientific and Virgo Collaborations), Phys. Rev. X 6, 041015 (2016); 8, 039903(E) (2018).

[3] B. P. Abbott et al. (LIGO Scientific and Virgo Collaborations), Astrophys. J. Lett. 882, L24 (2019).

[4] B. P. Abbott et al. (LIGO Scientific and Virgo Collaborations), Phys. Rev. X 9, 031040 (2019).

[5] S. Bird, I. Cholis, J. B. Muñoz, Y. Ali-Haïmoud, M. Kamionkowski, E. D. Kovetz, A. Raccanelli, and A. G. Riess, Phys. Rev. Lett. 116, 201301 (2016).

[6] M. Sasaki, T. Suyama, T. Tanaka, and S. Yokoyama, Classical Quantum Gravity 35, 063001 (2018).

[7] L. Barack et al., Classical Quantum Gravity 36, 143001 (2019).

[8] B. Carr, K. Kohri, Y. Sendouda, and J. Yokoyama, arXiv: 2002.12778.

[9] J. Garcìa-Bellido and S. Clesse, Phys. Dark Universe 19, 144 (2018).

[10] Y. Ali-Haïmoud, Phys. Rev. Lett. 121, 081304 (2018).

[11] V. Desjacques and A. Riotto, Phys. Rev. D 98, 123533 (2018).

[12] G. Ballesteros, P. D. Serpico, and M. Taoso, J. Cosmol. Astropart. Phys. 10 (2018) 043.

[13] A. Moradinezhad Dizgah, G. Franciolini, and A. Riotto, J. Cosmol. Astropart. Phys. 11 (2019) 001.

[14] V. De Luca, G. Franciolini, P. Pani, and A. Riotto, J. Cosmol. Astropart. Phys. 04 (2020) 052.

[15] M. Ricotti, Astrophys. J. 662, 53 (2007).

[16] M. Ricotti, J. P. Ostriker, and K. J. Mack, Astrophys. J. 680, 829 (2008).

[17] P. D. Serpico, V. Poulin, D. Inman, and K. Kohri, Phys. Rev. Research 2, 023204 (2020).

[18] J. R. Rice and B. Zhang, J. High Energy Astrophys. 13-14, 22 (2017).

[19] S. L. Shapiro and S. A. Teukolsky, Black Holes, White Dwarfs, and Neutron Stars: The Physics of Compact Objects (Wiley, New York, 1983).

[20] K. J. Mack, J. P. Ostriker, and M. Ricotti, Astrophys. J. 665, 1277 (2007).

[21] J. Adamek, C. T. Byrnes, M. Gosenca, and S. Hotchkiss, Phys. Rev. D 100, 023506 (2019).

[22] E. Bertschinger, Astrophys. J. Suppl. 58, 39 (1985).

[23] E. Barausse, V. Cardoso, and P. Pani, Phys. Rev. D 89, 104059 (2014).

[24] S. Oh and Z. Haiman, Mon. Not. R. Astron. Soc. 346, 456 (2003).

[25] G. Hasinger, J. Cosmol. Astropart. Phys. 07 (2020) 022.
[26] Y. Ali-Hamoud and M. Kamionkowski, Phys. Rev. D 95, 043534 (2017).

[27] Y. Ali-Hamoud, E. D. Kovetz, and M. Kamionkowski, Phys. Rev. D 96, 123523 (2017).

[28] G. Hütsi, M. Raidal, and H. Veermäe, Phys. Rev. D 100, 083016 (2019).

[29] D. Inman and Y. Ali-Haïmoud, Phys. Rev. D 100, 083528 (2019).

[30] M. Zumalacarregui and U. Seljak, Phys. Rev. Lett. 121, 141101 (2018).

[31] C. Alcock et al. (MACHO Collaboration), Astrophys. J. Suppl. 136, 439 (2001).

[32] R. A. Allsman et al. (MACHO Collaboration), Astrophys. J. Lett. 550, L169 (2001).

[33] M. Oguri, J. M. Diego, N. Kaiser, P. L. Kelly, and T. Broadhurst, Phys. Rev. D 97, 023518 (2018).

[34] P. N. Wilkinson, D. R. Henstock, I. W. A. Browne, A. G. Polatidis, P. Augusto, A. C. S. Readhead, T. J. Pearson, W. $\mathrm{Xu}, \mathrm{G}$. B. Taylor, and R. C. Vermeulen, Phys. Rev. Lett. 86, 584 (2001).

[35] D. Quinn, M. Wilkinson, M. Irwin, J. Marshall, A. Koch, and V. Belokurov, Mon. Not. R. Astron. Soc. 396, L11 (2009).

[36] T. D. Brandt, Astrophys. J. Lett. 824, L31 (2016).

[37] S. M. Koushiappas and A. Loeb, Phys. Rev. Lett. 119, 041102 (2017).

[38] R. Murgia, G. Scelfo, M. Viel, and A. Raccanelli, Phys. Rev. Lett. 123, 071102 (2019).

[39] D. Gaggero, G. Bertone, F. Calore, R. M. T. Connors, M. Lovell, S. Markoff, and E. Storm, Phys. Rev. Lett. 118, 241101 (2017).

[40] J. Manshanden, D. Gaggero, G. Bertone, R. M. T. Connors, and M. Ricotti, J. Cosmol. Astropart. Phys. 06 (2019) 026.

[41] Y. Inoue and A. Kusenko, J. Cosmol. Astropart. Phys. 10 (2017) 034.

[42] B. P. Abbott et al. (LIGO Scientific and Virgo Collaborations), Phys. Rev. Lett. 123, 161102 (2019).

[43] B. Carr, M. Raidal, T. Tenkanen, V. Vaskonen, and H. Veerme, Phys. Rev. D 96, 023514 (2017).

[44] N. Bellomo, J. L. Bernal, A. Raccanelli, and L. Verde, J. Cosmol. Astropart. Phys. 01 (2018) 004.

[45] S. Hild et al., Classical Quantum Gravity 28, 094013 (2011).

[46] L. Liu, Z. K. Guo, and R. G. Cai, Eur. Phys. J. C 79, 717 (2019).

[47] V. De Luca, G. Franciolini, P. Pani, and A. Riotto, J. Cosmol. Astropart. Phys. 06 (2020) 044.

[48] K. Inayoshi, E. Visbal, and Z. Haiman, arXiv:1911.05791. 